\title{
A Spectroscopic Search for White Dwarf Companions to 101 Nearby M Dwarfs*
}

\author{
Ira Bar $^{1}$, Paul Vreeswijk ${ }^{1}$ (1), Avishay Gal-Yam ${ }^{1}$ (1), Eran O. Ofek ${ }^{1}$ (1), and Gijs Nelemans ${ }^{2}$ \\ ${ }^{1}$ Benoziyo Center for Astrophysics, Faculty of Physics, The Weizmann Institute for Science, Rehovot 76100, Israel; ira7bar@gmail.com \\ ${ }^{2}$ Department of Astrophysics/IMAPP, Radboud University, P.O. Box 9010, NL-6500 GL Nijmegen, The Netherlands \\ Received 2017 March 21; revised 2017 October 1; accepted 2017 October 3; published 2017 November 14
}

\begin{abstract}
Recent studies of the stellar population in the solar neighborhood $(<20 \mathrm{pc})$ suggest that there are undetected white dwarfs (WDs) in multiple systems with main-sequence companions. Detecting these hidden stars and obtaining a more complete census of nearby WDs is important for our understanding of stellar and galactic evolution, as well as the study of explosive phenomena. In an attempt to uncover these hidden WDs, we present intermediate resolution spectroscopy over the wavelength range of 3000-25000 $\AA$ of 101 nearby M dwarfs (dMs), observed with the Very Large Telescope X-Shooter spectrograph. For each star we search for a hot component superimposed on the $\mathrm{dM}$ spectrum. X-Shooter has excellent blue sensitivity and thus can reveal a faint hot WD despite the brightness of its red companion. Visual examination shows no clear evidence of a WD in any of the spectra. We place upper limits on the effective temperatures of WDs that may still be hiding by fitting dM templates to the spectra and modeling the WD spectra. On average our survey is sensitive to WDs hotter than about $5300 \mathrm{~K}$. This suggests that the frequency of WD companions of $T_{\text {eff }} \gtrsim 5300 \mathrm{~K}$ with separation of the order of $\lesssim 50$ au among the local $\mathrm{dM}$ population is $<3 \%$ at the $95 \%$ confidence level. The reduced spectra are made available via the WISeREP ${ }^{3}$ repository.
\end{abstract}

Key words: binaries: general - stars: late-type - white dwarfs

Supporting material: figure set, machine-readable tables

\section{Introduction}

White dwarf (WD) stars are an important ingredient of stellar populations. As the end state of over $97 \%$ of stars (Fontaine et al. 2001), they play a crucial role in the understanding of stellar and galactic evolution. For example, relating the luminosity function of these stars to their cooling sequences can yield estimates of their age, and thus the age of the galactic disk and the universe (e.g., Winget \& van Horn 1987; GarcíaBerro \& Oswalt 2016).

Characterizing WDs in binary and higher multiplicity systems is paramount for many fields of research such as the WD mass-radius relation (Provencal et al. 1998), the WD initial-to-final mass relation (Weidemann 2000), and also explosive phenomena. For example, the origin of $\mathrm{SN}$ Ia explosions is still unknown. There is evidence that WDs are the progenitors of these explosions (Nugent et al. 2011), but the trigger of the explosion is still a puzzle. The two common models suggest a WD accreting material from a main-sequence or red giant companion, or two binary WDs merging and exploding (see Howell 2011 and Maoz et al. 2014 for reviews). Both models struggle to ignite an explosive detonation in simulations (Dong et al. 2015). A different model suggests an explosion due to a collision of two WDs in a triple system (Katz \& Dong 2012; Kushnir et al. 2013). This scenario easily produces explosions, but it is not clear whether enough systems such as these exist to account for the observed supernova rates. Improved statistics of WDs in multiple systems can constrain these models.

WDs are compact faint objects and are thus difficult to detect in multiple systems in which brighter, main-sequence

* Based on observations collected in service mode using the Very Large Telescope (VLT) under program IDs 095_D-0949(A) and 096_D-0963(A).

3 http://wiserep.weizmann.ac.il companions are present. Therefore, efforts have been made to obtain a complete census of WDs in the nearby solar neighborhood, from which statistics about galactic populations can be inferred. Holberg et al. made two such attempts for the local volume within $20 \mathrm{pc}$ (Holberg et al. 2002, 2008) reaching an estimated completeness of $80 \%$, suggesting there are still $\sim 33$ undetected WDs left in this volume. Further efforts including Sion et al. (2009), Giammichele et al. (2012), and Oswalt et al. (2016) have improved upon this, reaching an estimated 86\% completeness. In contrast, Katz et al. (2014) claims that the completeness fraction of Holberg et al. (2008) is over-estimated and that the actual value is smaller.

So are there "missing" WDs? The answer may hide in multiple systems. According to Oswalt et al. (2016), in the local neighborhood $74 \%$ of WDs are single stars and only $26 \%$ are in binary or higher multiplicity systems. This is in contrast with the progenitors of these WDs-main-sequence stars of K type and earlier (up to the minimum mass for supernova)which show multiplicity rates of $\sim 45 \%$ and higher (e.g., Mason et al. 2009; Raghavan et al. 2010; De Rosa et al. 2014).

Ferrario (2012) suggests that this discrepancy may be due to an observational bias-WDs in binaries are simply too faint compared to their companions, and are thus not detected. Katz et al. (2014) provided observational evidence supporting this claim using a WD luminosity-cooling age relation to derive the theoretically expected distribution of absolute visual magnitudes. They showed that the observed single WDs in the $20 \mathrm{pc}$ sample of Holberg et al. (2008) roughly follow this distribution, while the number of WDs in binaries drops compared to the expectation for magnitudes of 12 and fainter. This gap between expected and observed WDs in binaries implies that there are $\sim 100$ such nearby missing WDs, hiding in the light of main-sequence companions. This is a significant number, considering that the local $20 \mathrm{pc}$ volume contains $\sim 1900$ nonWD stars in total. 
We present an attempt to unveil some of those WD companions by obtaining spectra of 101 nearby $\mathbf{M}$ dwarfs using the Very Large Telescope X-Shooter spectrograph. The targets were selected based on their strong near-UV (NUV) emission as measured by the GALEX survey (Martin et al. 2005), which may arise from the contribution of a hidden WD to the spectrum, or from magnetic activity of the $\mathrm{dM}$. We attempt to detect WD companions by examining the spectra for characteristic features of WDs. As no such evidence of WD presence is visible, we then put upper limits on the effective temperatures of WDs that may still be hiding below our detection threshold.

This paper is organized as follows. In Section 2 we present the observations, the results and analysis are given in Section 3, and in Section 4 we discuss and summarize the main ideas.

\section{Data}

\subsection{Target Selection}

Our initial sample consists of dMs within $20 \mathrm{pc}$ of the Sun, taken from the Gliese Catalog of Nearby Stars, 3rd edition (Gliese \& Jahreiss 1995). We have selected dMs since earlier types would be too bright and blue to allow detection of a faint blue companion. We have limited our selection to dMs with absolute $V$ magnitudes of 10-16 mag. The faint limit was set by the faintest single WD in both the Holberg et al. (2008) and Gliese catalogs. Thus, a WD companion to a dM fainter than that would have to be brighter than the $\mathrm{dM}$ companion and would dominate the spectrum. In that case, the star would not be classified as a dM in the first place. The bright limit was set to ensure that dwarf stars were selected and not sub-giants, as the Gliese catalog does not always include the luminosity class of the $\mathrm{M}$ stars. In addition, we have discarded targets that have nearby objects in the catalog with separations of less than $30^{\prime \prime}$. To increase our chances of detection, we cross-correlated our target list with the GALEX survey, choosing the targets that showed the strongest NUV emission, compared to their $V$ magnitude ( $N U V-V$ color). This emission can be caused by the contribution of a hidden WD companion to the blue side of the spectrum. Jones \& West (2016) showed that dM-WD binaries tend to emit more NUV than single dMs, but also showed that this can also be the result of magnetic activity of dMs.

Cross matching between the Gliese and GALEX catalogs was done using tools in the astronomy $\&$ astrophysics package for Matlab (Ofek 2014). Figure 1 illustrates our color cut which left us with a total of $138 \mathrm{dMs}$.

\subsection{Observations and Data Reduction}

The observations were performed with the X-Shooter spectrograph (Vernet et al. 2011) mounted at the Kueyen unit of the Very Large Telescope (UT2), operated by the European Southern Observatory (ESO) on Cerro Paranal in Chile (Program IDs 095_D-0949(A) and 096_D-0963(A), PI: Paul Vreeswijk). The X-Shooter records spectra with three separate arms simultaneously-UVB, VIS, and NIR. This allows a wide wavelength range (3000-25000 ̊) while maintaining good sensitivity throughout the range. In particular, the instrument is very blue-sensitive down to the atmospheric cutoff around $3000 \AA$ A. Thus, we benefit from the ability to characterize the $\mathrm{dM}$ spectrum in the red part while allowing possible detection of WD contribution in the blue part.

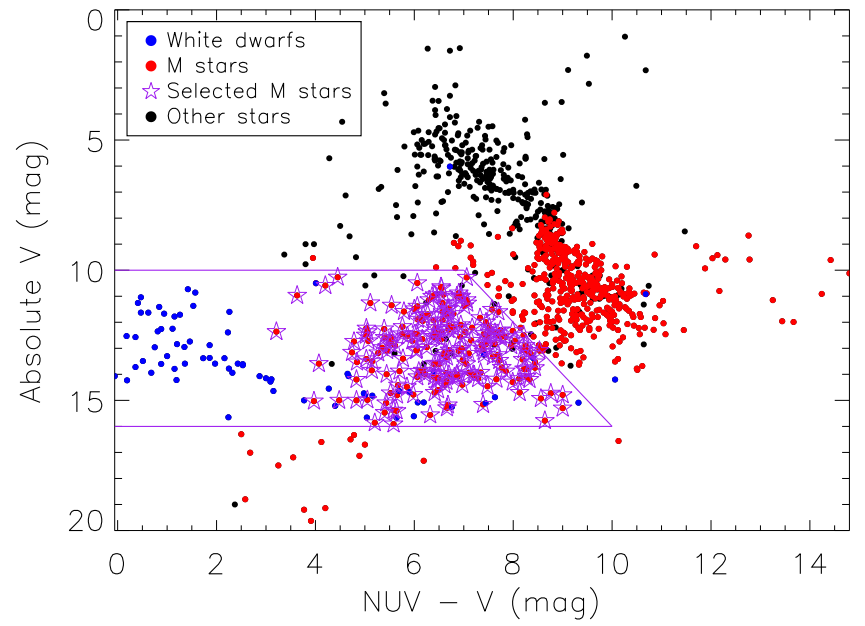

Figure 1. $V / N U V-V$ magnitude-color diagram of the stars in the Gliese catalog within $20 \mathrm{pc}$ of the Sun. $M$ stars are shown in red, single WDs (including additions from the Holberg et al. (2008) catalog) are shown in blue and other stars are shown in black. The purple line illustrates our color cut and the purple stars are the $138 \mathrm{M}$ stars out of which our targets were selected.

Since our targets are very bright, our observation plan was submitted to the ESO as a "filler" program, to be executed during poor seeing conditions $(>1$ !" 5$)$. We obtained servicemode observations with VLT/X-Shooter for 60 (41) targets during ESO Period 95 (96), securing spectra of a total of 101 dMs. Corrected locations of all targets were calculated using proper motions from the Stauffer et al. (2010) 2MASS-Gliese cross-correlation catalog. Exposure times were typically $120 \mathrm{~s}$ for UVB, $90 \mathrm{~s}$ for VIS, and four separate exposures of $30 \mathrm{~s}$ each for NIR. In some cases, exposure times were increased for faint targets or decreased to prevent saturation of the detector. Moreover, saturation was avoided for bright targets $\left(M_{V} \sim 10\right)$ by using a $1 \times 1$ binning read-out mode instead of $1 \times 2$. All of the observations were made in the Stare mode using the widest 5". 0 slit, to increase the chances of including a WD companion in the spectrum (up to a separation of 40 au for a $16 \mathrm{pc}$ target, the distance at half the $20 \mathrm{pc}$ volume). The resolving power of the X-Shooter for the 5!"0 slit was measured to be about 5000, 9000, and 5000 for the UVB, VIS, and NIR arms, respectively. This was done by examining several unresolved lines in the spectra and dividing their wavelengths by their full width at half maximum.

Spectra were reduced using the ESO's Reflex pipeline version 2.8 (Freudling et al. 2013). Automatic telluric correction was performed by the pipeline. A built-in "optimal extraction" algorithm (Horne 1986) was used to increase the signal-to-noise ratio $(\mathrm{S} / \mathrm{N})$. For the UVB and VIS arms, optimal extraction yielded a mean improvement of $5 \%$ and $4 \%$ in SNR, respectively, compared to standard extraction. On the other hand, for the NIR arm a mean $4 \%$ decrease in SNR was noted, with artifacts introduced to some of the spectra. Thus, we have decided to use optimal extraction for the UVB and VIS arms only, and standard extraction for the NIR arm.

We removed very noisy parts in the UVB and VIS arms below 3200 and $5500 \AA$, respectively. The three spectral pieces were then stitched together using overlap regions as a reference. Both the UVB and the VIS spectra include a feature at $\sim 5500-5800 \AA$ due to the dichroic splitter of the X-Shooter (Chen et al. 2014). Thus, we excluded this feature from the overlap regions. 


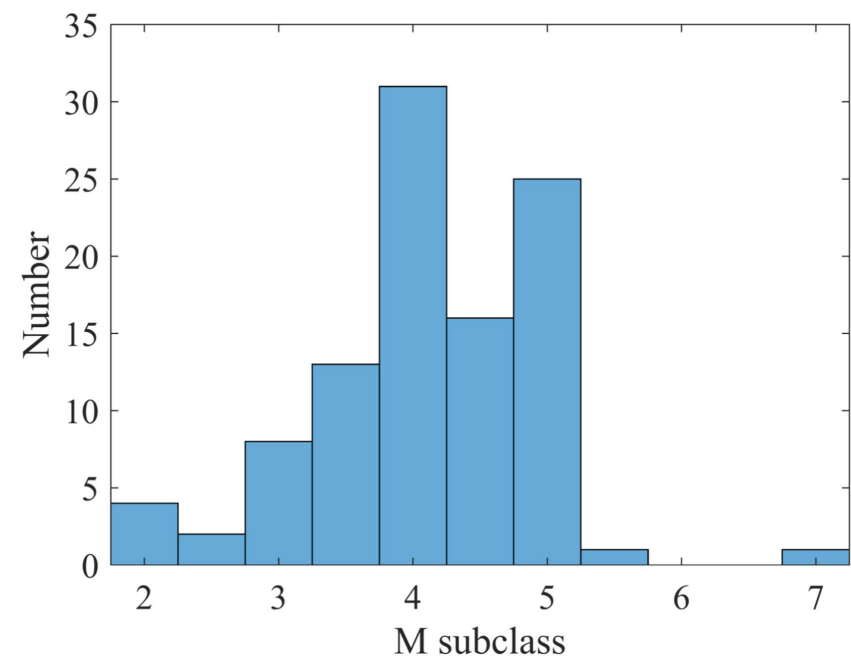

Figure 2. The M subclass distribution of the stars observed in this study.

The pipeline produces a flux-calibrated spectrum for each target using a spectroscopic standard star that was observed on the same night. This procedure assumes photometric nights. Therefore, we performed absolute calibration for each object using JHK band photometry from the 2MASS catalog (Skrutskie et al. 2006), since these are the only bands that are reported for all of our targets in the SIMBAD database (Wenger et al. 2000). We calculated JHK synthetic photometry for each spectrum and rescaled the flux to match the photometric data.

The log of spectroscopic observations is presented in Table 2 in the Appendix, along with plots of the reduced spectra. These are also available on the WISeREP ${ }^{4}$ repository (Yaron \& GalYam 2012) and are searchable via object name or by the type of "M dwarf." The subclass distribution of our sample is presented in Figure 2.

\section{Results and Analysis}

\subsection{Activity and Multiplicity}

Out of the 101 observed targets, 65 of them show strong emission lines that are indicative of magnetic activity: the hydrogen Balmer series and the Ca II $\mathrm{H}$ and $\mathrm{K}$ lines (see Reid \& Hawley 2005). An example of these lines for target GJ2069 is shown in Figure 3. The remainder of the targets show little or no emission lines. Five spectra are showing double emission lines (also shown in Figure 3), which suggests that these are in fact binary stars which are not listed in the Gliese catalog. A search of binary star catalogs (Mason et al. 2001 and Shkolnik et al. 2010) shows that these are indeed known binary or higher multiplicity systems, and revealed 21 additional multiples in our sample with separations smaller than half of our slit width (targets were positioned in the center of the slit) that were not filtered out initially. The separations of these systems are listed in Table 1 and we give special care to these in our analysis later on.

\subsection{White Dwarf Spectral Features}

According to the Kleinman et al. (2013) SDSS survey, over $90 \%$ of WDs are classified as having either hydrogen or helium

\footnotetext{
http://wiserep.weizmann.ac.il
}

atmospheres, indicating a spectral type of DA or DB, respectively. Thus, we first visually examine the spectrum of each target in search of distinct broad absorption features typical of these spectroscopic classes. For type DA, these would be the hydrogen Balmer lines at 6563, 4861, 4341, and $3970 \AA$. For type DB, the dominant lines are from neutral helium at 4026, 4471, and $5875 \AA$ (Bergeron et al. 2011). Some WDs show only a continuum spectrum-type DC. According to Giammichele et al. (2012), those can be hydrogen atmosphere WDs of $T_{\text {eff }} \leqslant 5000 \mathrm{~K}$ or helium atmosphere WDs of $T_{\text {eff }} \leqslant 12,000 \mathrm{~K}$. The search for this type and other more exotic types was done by looking for a clear rise in the blue part of each target. No evidence for DA or DB features, nor of a clear blue rise, was found in any of the spectra.

\subsection{Limits on the Temperature of Unseen WDs}

Next, we place upper limits on the effective temperature of WDs that may still be hiding below our detection threshold. We do so by modeling spectra of WDs with a mass of $0.6 M_{\odot}$ as blackbodies of varying effective temperatures. We use this mass value as it is the peak of the narrow observed WD mass distribution (see Bergeron et al. 2001; Kepler et al. 2007). Using these models and spectral templates for each dM, we determine at which temperature the WD models can be rejected.

As spectral dM templates, we tried to use the Pickles (1998) spectral library, which did not provide good enough fits to our data due to the low resolution $(\sim 500 \AA)$ and to a limited number of spectral subtypes. We have also tried using the PHOENIX synthetic spectral models (Husser et al. 2013), which did not describe our data well in the UVB and NIR parts. Eventually, we have decided to use our own data as templates, under the assumption that most of our targets do not hide WD companions. Indeed, the self-template method yields better fits for the vast majority of our targets (see Figure 6), compared with the alternative methods.

The best fitting template for each target was determined as the one with the lowest Residual Sum of Squares (RSS) score

$$
\operatorname{RSS}=\sum_{i}\left(f_{S, i}-f_{T, i}\right)^{2},
$$

where $f_{S, i}, f_{T, i}$ are the flux values of the current target and of each template, respectively. Before calculating the RSS score, the spectra were rescaled to each other such that the flux integral is equal to one. We excluded from the fit the blue part below $6300 \AA$ to minimize possible WD contamination, while keeping the prominent $\mathrm{dM}$ features that extend to redder wavelengths. We also excluded wavelength ranges of known

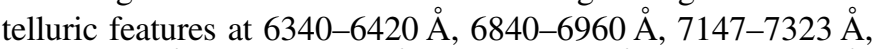

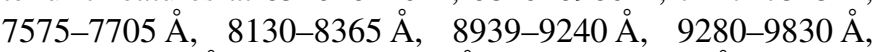
10810-11710 А, 12670-12710 А, 13000-15030 А, 17350$19810 \AA, \quad 19950-20350 \AA$, and 20480-20820 et al. 2014). As we only have 101 spectra to work with, dividing them into magnetically active and non-active would limit our ability to find good templates. Thus, we included both active and non-active stars in the template bank for all targets while excluding the Balmer $\mathrm{H} \alpha$ line from the fit range. As shown, for example, in Figure 6, nice fits are produced also when using active templates for non-active targets and vice versa. 


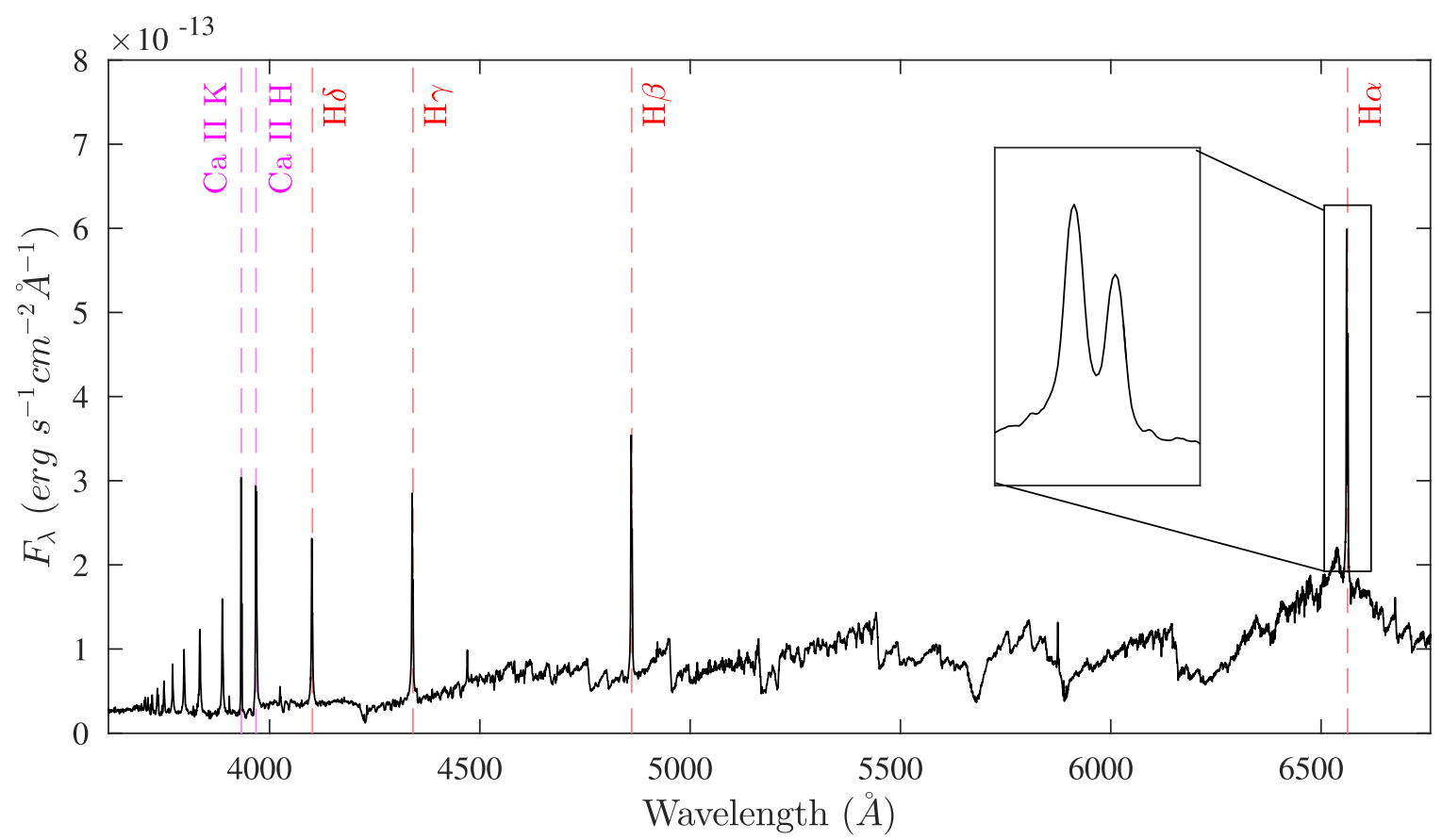

Figure 3. Example emission lines for GJ2069. The red lines indicate the first four Balmer series lines and the pink line indicate the Ca IIH/K lines. The double H $\alpha$ line is shown in the inset.

As WD synthetic spectra were not available to us, we chose to model WDs as blackbodies of varying effective temperatures and with radii that fit a typical mass of $0.6 M_{\odot}$. The radius for each $T_{\text {eff }}$ value was calculated using $R^{2}=G M / g$ and the surface gravity $(\log g)$ values from the publicly available WD color model grids of Holberg \& Bergeron (2006), Kowalski \& Saumon (2006), Tremblay et al. (2011), and Bergeron et al. (2011). The absolute flux values of the models were calculated using these radii and the distance to each target, obtained from the Gliese catalog.

Next, we determine the hottest WD that may be hiding in the data for each target. The dM fits are not perfect and exhibit correlated residuals (i.e., spectral regions that are systematically lower or higher between each target and its template). Such correlated noise make it very difficult to make quantitative statements. We attempt to explain the observed spectrum using a combination of the template spectrum and an approximate WD model, and to ask when the temperature of the WD produces a "noticeable" effect on the composed spectrum. In each such fit, the WD flux is completely determined by its radius (assuming a $0.6 M_{\odot} \mathrm{WD}$ ), its distance, and assuming a blackbody emitter. Therefore, we use several methods to put limits on the WD temperature that can be hidden in each system. We note that since the WD luminosity is very sensitive to its effective temperature (i.e., $\propto T_{\text {eff }}^{4}$ ), any reasonable estimator will yield similar results regardless of the details of the test. In the first method, we reject WD models that yield an RSS score double than that of the template alone. We add the flux of the best fit template to the blackbody models for a grid of effective temperatures between 1500 and $20,000 \mathrm{~K}$, and calculate the RSS score for each temperature. We then compare these scores with the RSS of the fit with no WD. The lowest temperature for which the RSS score is more than double the score without the model is set as our upper limit. This test is arbitrary, but due to the extreme sensitivity of the luminosity on $T_{\text {eff }}$, the obtained limits are similar for different criteria. For example, changing the threshold to three times the RSS yields an average increase in the limit temperature of only $400 \mathrm{~K}$. Our temperature grid follows that of the color models-from 1500 to $5500 \mathrm{~K}$ in steps of $250 \mathrm{~K}$ and then to $15,000 \mathrm{~K}$ in steps of $500 \mathrm{~K}$. As opposed to the template fitting, we now calculate the RSS score for wavelengths $3200-10000 \AA$, where WD contribution would be dominant. In addition to the $\mathrm{H} \alpha$ line and tellurics, we also remove from the fit range the rest of the Balmer emission lines, the Ca II lines, and the X-Shooter dichroic feature.

In the second method, we reject WD models that exceed the fit residuals envelope. The envelope is defined as the 99th percentile of the flux for the absolute value of the fit residuals. In this case, the limit is set as the lowest temperature for which the blackbody model flux exceeds the envelope. In other words, the rejected WD model is the coldest one, which is not consistent with the residuals of the fit.

The third method is robust and template-independent. Here, we compare the integrated flux of the very blue part of the $\mathrm{dM}$ spectra to that of our blackbody models. The limit is set as the temperature of the coldest model for which the total blue flux is greater than that of the $\mathrm{dM}$. This was done for wavelengths of $3200-3700 \AA$, as this range features only a weak continuum from the dMs. In addition, this range is bluer than that of the Balmer series, thus avoiding the typical absorption features of WDs and ensuring that blackbody is a good approximation for WD spectra. An example of the three methods is given in Figure 4.

To verify that the blackbody model describes WDs well enough for our purposes, we repeat the analysis using explicit WD models from Koester (2010). Since the publicly available spectra $^{5}$ range from $T_{\text {eff }} \geqslant 6000 \mathrm{~K}$, we performed the analysis on a sample of targets, which yielded higher limits than this

\footnotetext{
5 http://svo2.cab.inta-csic.es/theory/newov/index.php?model=koester
} 
Table 1

Obtained Results of Spectral Analysis

\begin{tabular}{|c|c|c|c|c|c|c|}
\hline Name & Template & $\begin{array}{l}\text { RSS } \\
(\mathrm{K})\end{array}$ & $\begin{array}{l}\text { Env. } \\
(\mathrm{K})\end{array}$ & $\begin{array}{l}\text { Blue Flux } \\
\text { (K) }\end{array}$ & Act. $^{a}$ & $\begin{array}{l}\text { Sep. }{ }^{b} \\
\left({ }^{\prime \prime}\right)\end{array}$ \\
\hline NN3010 & .. & & & 5250 & $\mathrm{~V}$ & 0.1 \\
\hline NN3017 & GJ2021 & 5250 & 6500 & 5250 & V & $\ldots$ \\
\hline NN3027 & NN3682 & 5250 & 6500 & 5500 & $\mathrm{~V}$ & $\ldots$ \\
\hline NN3033 & NN3518 & 5000 & 6500 & 4750 & V & $\ldots$ \\
\hline NN3034 & GJ1204 & 5000 & 6500 & 5000 & $\cdots$ & $\cdots$ \\
\hline NN3039 & $\cdots$ & $\cdots$ & $\cdots$ & 6000 & V & 0.7 \\
\hline GJ1019 & NN3227 & 6000 & 6000 & 5000 & $\cdots$ & $\cdots$ \\
\hline NN3056 & NN3142 & 4500 & 6000 & 5250 & $\ldots$ & $\ldots$ \\
\hline GJ1024 & GJ1031 & 6000 & 7000 & 5500 & $\ldots$ & $\ldots$ \\
\hline GJ1029 & NN3937 & 4750 & 6000 & 3750 & $\ldots$ & $\ldots$ \\
\hline GJ1031 & NN3225 & 5000 & 5500 & 5250 & V & $\ldots$ \\
\hline GJ2021 & GJ1031 & 4500 & 5500 & 4750 & $\ldots$ & $\ldots$ \\
\hline NN3076 & $\ldots$ & $\ldots$ & $\ldots$ & 4250 & V & 0.3 \\
\hline NN3119 & NN4292 & 4000 & 5000 & 4250 & V & $\ldots$ \\
\hline Gl83.1 & NN3225 & 4000 & 5000 & 4750 & V & $\ldots$ \\
\hline NN3129 & $\ldots$ & $\ldots$ & $\ldots$ & 5500 & $D^{c}$ & $\ldots$ \\
\hline NN3142 & NN3149 & 5250 & 7000 & 5500 & V & $\cdots$ \\
\hline NN3143 & G1828 & 6000 & 6500 & 5500 & V & $\cdots$ \\
\hline NN3149 & GJ1031 & 6000 & 7000 & 6500 & V & $\ldots$ \\
\hline NN3148 & GJ1284 & 6500 & 8000 & 6500 & V & $\cdots$ \\
\hline Gl102 & GJ1031 & 5250 & 6500 & 5250 & $\ldots$ & $\ldots$ \\
\hline GJ1055 & NN3253 & 3750 & 5000 & 4250 & $\ldots$ & $\ldots$ \\
\hline NN3224 & $\ldots$ & $\ldots$ & $\ldots$ & 4500 & D & $0.3+2$ \\
\hline NN3225 & Gl83.1 & 4500 & 5250 & 4000 & $\mathrm{~V}$ & $\ldots$ \\
\hline NN3227 & Gl729 & 7500 & 7000 & 6000 & $\mathrm{~V}$ & $\ldots$ \\
\hline NN3237 & NN3253 & 6500 & 6500 & 5500 & V & $\ldots$ \\
\hline NN3253 & NN3237 & 4500 & 5500 & 4500 & $\cdots$ & $\ldots$ \\
\hline NN3261 & $\ldots$ & $\ldots$ & $\ldots$ & 6500 & $\mathrm{~V}$ & 0.8 \\
\hline NN3296 & NN3225 & 5500 & 6000 & 5000 & V & $\ldots$ \\
\hline NN3304 & $\cdots$ & $\cdots$ & $\cdots$ & 5500 & V & 1.1 \\
\hline NN3322 & $\ldots$ & $\ldots$ & $\ldots$ & 6500 & V & 1.4 \\
\hline NN3326 & NN3967 & 6000 & 6500 & 5250 & V & $\ldots$ \\
\hline NN3332 & $\ldots$ & $\ldots$ & $\ldots$ & 8000 & $\mathrm{~V}$ & 0.8 \\
\hline NN3344 & G1207.1 & 10000 & 9500 & 8500 & & $\ldots$ \\
\hline G1207.1 & GJ1284 & 10500 & 9500 & 8000 & V & $\ldots$ \\
\hline GJ1083 & $\ldots$ & $\ldots$ & $\ldots$ & 4000 & $\mathrm{~V}$ & 0.5 \\
\hline NN3405 & G1828 & 6500 & 7500 & 6000 & $\ldots$ & $\ldots$ \\
\hline GJ1093 & GJ1286 & 4000 & 5250 & 3750 & $\ldots$ & $\ldots$ \\
\hline NN3423 & G1729 & 5500 & 6500 & 5500 & $\mathrm{~V}$ & $\ldots$ \\
\hline GJ1096 & NN3225 & 5000 & 6000 & 4500 & V & $\ldots$ \\
\hline Gl268.3 & $\cdots$ & $\cdots$ & $\cdots$ & 6500 & & 0.1 \\
\hline NN3454 & $\ldots$ & $\cdots$ & $\cdots$ & 4750 & V & 0.3 \\
\hline NN3463 & GJ1103 & 4250 & 5250 & 4750 & $\cdots$ & $\cdots$ \\
\hline GJ1103 & NN3463 & 5500 & 5500 & 5000 & $\cdots$ & $\ldots$ \\
\hline NN3466 & $\ldots$ & $\ldots$ & $\ldots$ & 5500 & & 1 \\
\hline GJ1108 & $\ldots$ & $\ldots$ & $\ldots$ & 7500 & $\mathrm{D}$ & $0.3+14$ \\
\hline Gl300 & $\ldots$ & $\ldots$ & $\ldots$ & 5250 & $\ldots$ & 2 \\
\hline NN3503 & NN3518 & 4500 & 5500 & 4250 & $\ldots$ & $\ldots$ \\
\hline GJ2069 & $\cdots$ & $\ldots$ & $\cdots$ & 7000 & $\mathrm{D}$ & $1+10+22$ \\
\hline Gl324 & NN3967 & 6500 & 7000 & 5500 & $\ldots$ & $\ldots$ \\
\hline NN3518 & NN3033 & 5000 & 6000 & 5000 & V & $\ldots$ \\
\hline GJ1116 & $\cdots$ & $\ldots$ & $\ldots$ & 3750 & V & 1.8 \\
\hline NN3543 & NN3344 & 8500 & 8000 & 6500 & $\mathrm{Ca}$ & $\cdots$ \\
\hline NN3549 & NN3937 & 4000 & 5250 & 4250 & V & $\cdots$ \\
\hline Gl347 & GJ1031 & 5250 & 6000 & 4500 & $\cdots$ & $\cdots$ \\
\hline Gl359 & G1347 & 4500 & 6000 & 4500 & $\ldots$ & $\ldots$ \\
\hline NN3571 & G1347 & 4750 & 6000 & 4750 & $\mathrm{~V}$ & $\ldots$ \\
\hline NN3572 & GJ1186 & 8500 & 8000 & 6500 & $\ldots$ & $\ldots$ \\
\hline NN3573 & NN3571 & 7000 & 7500 & 5000 & $\ldots$ & $\ldots$ \\
\hline NN3590 & GJ1186 & 6000 & 7500 & 5000 & $\ldots$ & $\ldots$ \\
\hline NN3647 & GJ1031 & 6000 & 8000 & 6500 & $\cdots$ & $\ldots$ \\
\hline NN3654 & Gl781.1 & 7000 & 7500 & 5500 & $\ldots$ & $\ldots$ \\
\hline NN3657 & GJ1031 & 4250 & 6000 & 4250 & $\ldots$ & $\ldots$ \\
\hline
\end{tabular}

Table 1

(Continued)

\begin{tabular}{|c|c|c|c|c|c|c|}
\hline Name & Template & $\begin{array}{l}\text { RSS } \\
(\mathrm{K})\end{array}$ & $\begin{array}{l}\text { Env. } \\
(\mathrm{K})\end{array}$ & $\begin{array}{l}\text { Blue Flux } \\
\text { (K) }\end{array}$ & Act. $^{a}$ & $\begin{array}{c}\text { Sep. } \\
\left({ }^{\prime \prime}\right)\end{array}$ \\
\hline NN3668 & NN3463 & 4500 & 6000 & 4750 & $\ldots$ & $\ldots$ \\
\hline NN3682 & NN3780 & 6000 & 6500 & 6000 & V & $\ldots$ \\
\hline NN3685 & NN3149 & 6500 & 7000 & 6000 & $\mathrm{~V}$ & $\ldots$ \\
\hline GJ1154 & NN3149 & 4250 & 5500 & 4750 & V & $\ldots$ \\
\hline NN3780 & NN3682 & 6000 & 6500 & 6000 & V & $\ldots$ \\
\hline GJ1179 & NN3682 & 4750 & 5250 & 3750 & V & $\ldots$ \\
\hline NN3808 & GJ2021 & 6000 & 6500 & 5000 & V & $\ldots$ \\
\hline Gl540.2 & NN4292 & 5500 & 6500 & 5250 & V & $\ldots$ \\
\hline NN3856 & NN3780 & 5250 & 6500 & 5500 & V & $\ldots$ \\
\hline GJ1186 & NN4292 & 5250 & 5500 & 4500 & $\ldots$ & $\ldots$ \\
\hline NN3900 & G1828 & 5500 & 7000 & 6000 & $\ldots$ & $\ldots$ \\
\hline NN3937 & GJ1029 & 4750 & 5500 & 4250 & $\ldots$ & $\ldots$ \\
\hline GJ1204 & GJ1284 & 6000 & 6500 & 5250 & V & $\ldots$ \\
\hline NN3967 & NN3326 & 4500 & 6000 & 5000 & V & $\ldots$ \\
\hline NN3981 & $\cdots$ & $\ldots$ & $\ldots$ & 5500 & V & 0.6 \\
\hline GJ1210 & $\ldots$ & $\ldots$ & $\ldots$ & 4750 & V & 0.4 \\
\hline NN4032 & NN3227 & 8500 & 8000 & 6500 & $\ldots$ & $\ldots$ \\
\hline NN4071 & NN3423 & 6000 & 7000 & 5500 & V & $\ldots$ \\
\hline G1729 & NN3227 & 4750 & 6000 & 5250 & V & $\ldots$ \\
\hline Gl781.1 & NN4279 & 7500 & 8000 & 7000 & V & $\ldots$ \\
\hline Gl791.2 & $\ldots$ & $\ldots$ & $\ldots$ & 5000 & V & $0+0.2$ \\
\hline Gl828 & NN3143 & 4750 & 6500 & 5500 & $\ldots$ & $\ldots$ \\
\hline NN4201 & $\cdots$ & $\cdots$ & $\cdots$ & 5500 & V & 0.8 \\
\hline G1836 & NN4071 & 6000 & 6500 & 6000 & $\ldots$ & $\ldots$ \\
\hline NN4215 & NN4292 & 4500 & 5500 & 4500 & $\ldots$ & $\ldots$ \\
\hline NN4231 & $\ldots$ & $\ldots$ & $\ldots$ & 7500 & V & $0.2+0.7$ \\
\hline NN4239 & NN3657 & 5250 & 6000 & 4500 & $\ldots$ & $\ldots$ \\
\hline NN4274 & GJ1154 & 4750 & 5250 & 4750 & V & $\ldots$ \\
\hline NN4279 & G1781.1 & 5000 & 6000 & 5500 & V & $\ldots$ \\
\hline NN4282 & $\ldots$ & $\ldots$ & $\ldots$ & 7500 & V & 1.5 \\
\hline NN4292 & NN3119 & 5500 & 5500 & 4500 & V & $\ldots$ \\
\hline NN4302 & G1781.1 & 6000 & 6500 & 5500 & $\ldots$ & $\ldots$ \\
\hline NN4326 & & & & 6500 & V & 0.1 \\
\hline GJ1284 & Gl83.1 & 9500 & 9000 & 7500 & V & $\cdots$ \\
\hline GJ1286 & G1207.1 & 5000 & 5250 & 4000 & $\ldots$ & $\ldots$ \\
\hline NN4360 & $\cdots$ & $\ldots$ & $\ldots$ & 4500 & $\mathrm{~V}$ & 0.6 \\
\hline NN4362 & GJ1284 & 7500 & 8000 & 7000 & V & $\ldots$ \\
\hline NN4378 & $\ldots$ & $\ldots$ & $\ldots$ & 7000 & D & $0.6+20$ \\
\hline
\end{tabular}

Notes. RSS, envelope, and blue flux are the limits obtained using the three methods. Targets without reported templates and RSS/envelope limits are those defined as close binaries.

${ }^{a}$ Activity $-\mathrm{B}+\mathrm{Ca}$ stands for Balmer and $\mathrm{Ca}$ II emission lines, $\mathrm{Ca}$ for $\mathrm{Ca}$ II lines only, D for double emission lines (Balmer and Ca II).

${ }^{\mathrm{b}}$ Companion separations from the WDS catalog, listed for targets where at least one companion is closer than 2 ." 5 .

${ }^{\mathrm{c}}$ Listed as binary in Shkolnik et al. (2010), no separation reported.

(This table is available in machine-readable form.)

temperature and found consistent results to within $500 \mathrm{~K}$, lending support to the use of the simple blackbody model.

As noted before, 26 of our targets are listed in binary catalogs as binaries or higher multiplicity systems with separations of less than 2!"5 (close binaries, hereafter), which is within half the X-Shooter slit width that we have used. Five of those display double emission lines in their spectra. The spectra of the rest of these close binaries may or may not contain more than one star. Thus, they are problematic for our limit analysis and must be analyzed with care. As a first precaution, we have taken these targets out of the template 

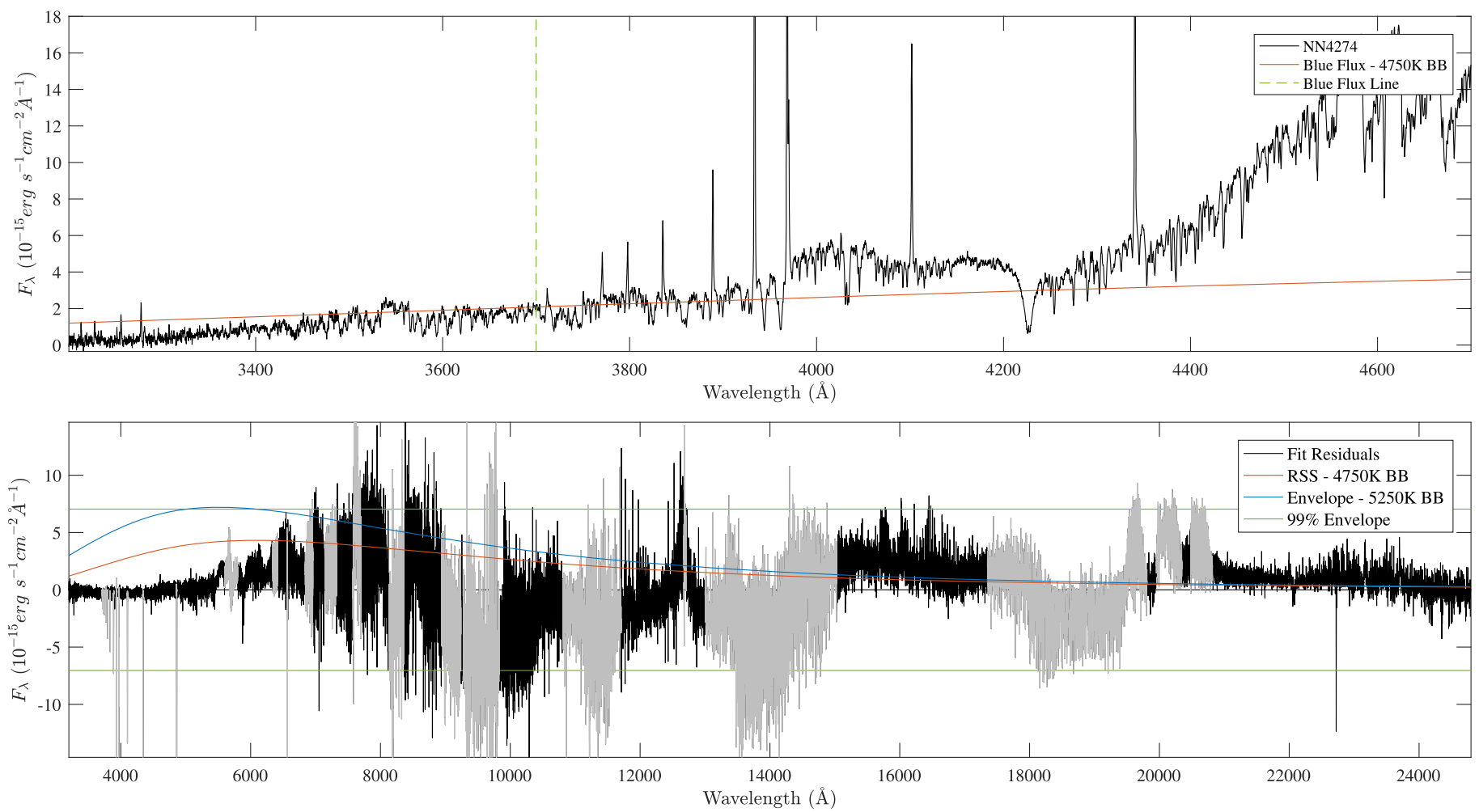

Figure 4. Example of the limit calculations for NN4274. Top panel: the blue part of the spectrum is shown in black. The rejected blackbody model from the blue flux method is shown in orange. The $3700 \AA$ line defining the blue flux region is shown in green. Bottom panel: the fit residuals from the best fit template GJ1154 are shown in black. In orange and blue are the rejected blackbody models from the RSS and envelope methods, respectively. The $99 \%$ flux envelope is shown in green.

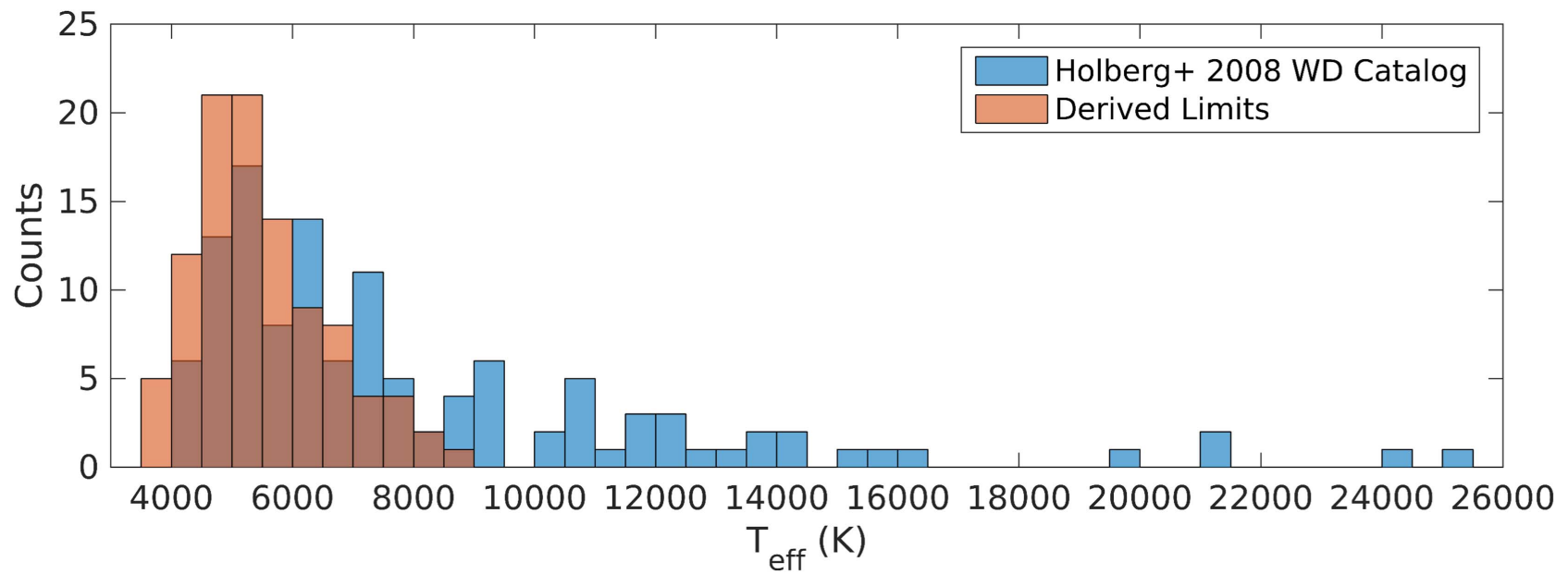

Figure 5. The effective temperature distributions of the limits derived in this study and of the WDs in the Holberg et al. (2008) catalog.

bank. Second, for these cases we only report the limits obtained using the blue flux method, which is independent of template.

The limits obtained and best fit templates for each target are listed in Table 1. The figure set for Figure 6 displays, for each target,

1. the spectrum,

2. the best fit template, rescaled to the flux of the target and

3. the residuals of the fit and the rejected blackbody models used to derive the limits.

The figure set for Figure 7 displays the spectra of the close binary targets, for which no template analysis was performed.

\section{Discussion}

Motivated by the evidence for missing WDs in multiple systems, we have used the X-Shooter to record spectra of 101 dMs that show strong NUV emission. We have examined these spectra for evidence of WD spectral features. When those were not found, we have used the spectra themselves as templates, modeled WDs as blackbodies, and obtained upper limits for the effective temperatures of WDs that may still be hidden, using three different methods. It is important to note that the limits obtained are only within our slit width of $5^{\prime \prime}$. Since our targets are positioned roughly at the center of the slit, this corresponds to a companion at $40 \mathrm{au}$ for a target at $16 \mathrm{pc}$ (the distance at half 


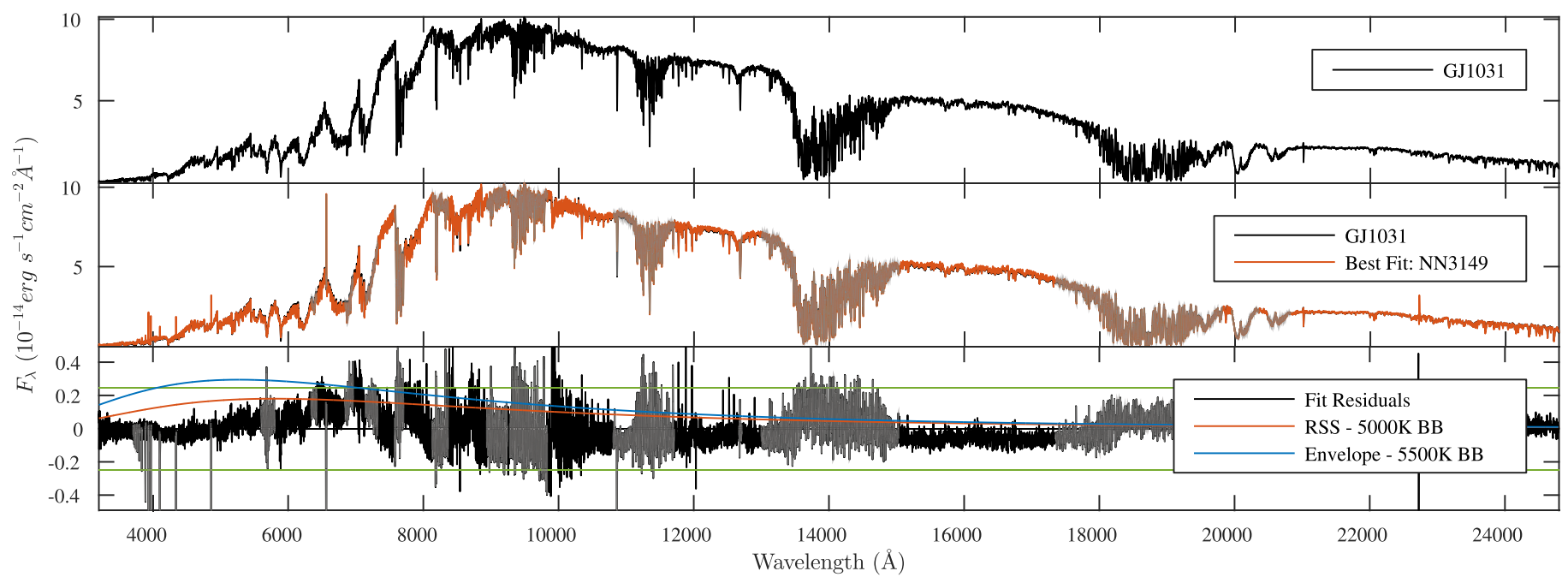

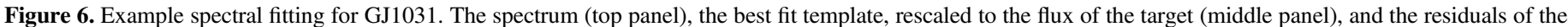

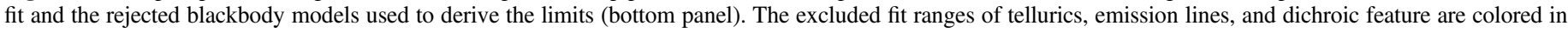
gray.

(The complete figure set (75 images) is available.)

the $20 \mathrm{pc}$ volume). Though our slit is only $5^{\prime \prime}$ wide, it is $11^{\prime \prime}$ long, which could allow a companion at larger separations. Thus, we take the order of $\sim 50$ au as our typical separation limit.

Figure 5 shows the effective temperature distribution of our derived limits, selected as the tightest limit for each target among the three methods. Also shown is the effective temperature distribution of WDs in the Holberg et al. (2008) catalog. According to these, our limit analysis is sensitive to $\sim 75 \%$ of WDs in the local volume. The average obtained limit for our $101 \mathrm{dM}$ sample is $5300 \mathrm{~K}$. Thus, the frequency of dMWD binaries with WDs hotter than that is smaller than $3 \%$ at the $95 \%$ confidence level.

The Gliese catalog lists $\sim 1900$ non-WD stars with distances below $20 \mathrm{pc}$. Assuming that $100 \mathrm{WD}$ companions are missing from that volume (Katz et al. 2014) and that WDs do not prefer specific class of companions, then $5 \%$ of the stars should have WD companions. As we have detected no WDs in our $101 \mathrm{dM}$ sample, this hypothesis can be rejected at the $99.3 \%$ confidence level. However, our survey is sensitive to WDs hotter than $\sim 5300 \mathrm{~K}$, corresponding to $\sim 75 \%$ of the known local population. In addition, our slit width limits our survey to separations of the order of $\lesssim 50 \mathrm{au}$, which corresponds to $55 \%$ of dM-WD pairs (Farihi et al. 2010). Taking both into account, the expectancy is to detect only two WDs in our sample, and this hypothesis can be rejected at the $86 \%$ confidence level. The missing WDs may be colder than our detection limit or outside our slit, thus undetected. Another possibility is that the missing WDs are not hidden in the shadow of dMs, but rather are companions to stars of earlier types, as proposed in Ferrario (2012). Or else, perhaps not as many WDs are missing from the local volume as claimed.

It is interesting to note that selecting targets according to NUV emission produced a strong bias toward active dMs. For example, for spectral types M4 and M5, which make up the majority of our sample, we noted a $62 \%$ activity fraction, as opposed to $\sim 35 \%$ for the SDSS survey of West et al. (2004). Studies of magnetic activity of dMs can thus benefit from our data set.
As mentioned before, our spectra are available online for public use. In our observations, we covered the entire wavelength range of active and non-active dMs at medium resolution, including several binaries with resolved double emission lines. We hope that this data will be useful for future research.

We would like to thank Barak Zackay for many insightful discussions. This work used the astronomy \& astrophysics package for Matlab (Ofek 2014), the SIMBAD database, operated at CDS, Strasbourg, France, and used data obtained from the Weizmann interactive supernova data repository (http://wiserep. weizmann.ac.il). This work also made use of the data products from the 2MASS, which is a joint project of the University of Massachusetts and the Infrared Processing and Analysis Center/ California Institute of Technology, funded by NASA and the NSF. This work has made use of the Washington Double Star Catalog maintained at the U.S. Naval Observatory, made use of the tables for evolutionary sequences of WD atmospheres of Holberg \& Bergeron (2006), Kowalski \& Saumon (2006), Tremblay et al. (2011), and Bergeron et al. (2011; http://www. astro.umontreal.ca/ bergeron/CoolingModels/), and made use of the observations made with the NASA Galaxy Evolution Explorer. GALEX is operated for NASA by the California Institute of Technology under NASA contract NAS5-98034. We acknowledge support from the Weizmann Institute, in particular the Kimmel Award.

\section{Appendix}

The appendix contains Table 2 of the observation log, Figure 6, which contains the spectra, the best fit templates, the fit residuals, and blackbody models of the derived limits. Excluded fit ranges of tellurics, emission lines, and dichroic feature are colored in gray. Also included is Figure 7, which displays the spectra of the close binary targets. 
Table 2

Observed M dwarfs

\begin{tabular}{|c|c|c|c|c|c|c|c|c|c|c|c|c|}
\hline Name & R.A. & Decl. & $\begin{array}{l}\text { Spectral } \\
\text { Type }\end{array}$ & $M_{V}$ & $\begin{array}{l}\text { Distance } \\
(\mathrm{pc})\end{array}$ & $\begin{array}{l}\text { Observation } \\
\text { Date }\end{array}$ & $\begin{array}{l}\text { Observation } \\
\text { UT Time }\end{array}$ & Airmass & $\begin{array}{c}\text { Seeing } \\
\left({ }^{\prime \prime}\right)\end{array}$ & $\begin{array}{l}\text { UVB Exposure } \\
\text { (s) }\end{array}$ & $\begin{array}{l}\text { VIS Exposure } \\
\text { (s) }\end{array}$ & $\begin{array}{l}\text { NIR Exposure } \\
\text { (s) }\end{array}$ \\
\hline NN3010 & 00:08:53.95 & $+20: 50: 22.4$ & M5 & 13.54 & 10.64 & 2015 Jul 16 & 09:54:56 & 1.452 & 0.9 & 120 & 90 & $4 \times 30$ \\
\hline NN3017 & $00: 15: 36.88$ & $-29: 46: 02.7$ & M4 & 14.31 & 17.86 & 2015 Aug 13 & $05: 25: 54$ & 1.138 & 1.5 & 120 & 90 & $4 \times 30$ \\
\hline NN3027 & $00: 18: 54.11$ & $+27: 48: 47.4$ & M4 & 13.86 & 19.23 & 2015 Oct 30 & $00: 41: 26$ & 1.914 & 1.2 & 120 & 90 & $4 \times 30$ \\
\hline NN3033 & $00: 24: 35.78$ & $+30: 02: 30.2$ & M5 & 14.54 & 18.87 & 2015 Oct 30 & 01:03:07 & 1.937 & 1.1 & 120 & 90 & $4 \times 30$ \\
\hline NN3034 & $00: 25: 20.42$ & $+22: 53: 04.0$ & M4 & 14.30 & 18.87 & 2015 Jun 20 & 10:06:16 & 1.566 & 1.1 & 120 & 90 & $4 \times 30$ \\
\hline NN3039 & $00: 32: 34.97$ & $+07: 29: 25.3$ & M4 & 12.70 & 11.63 & 2015 Jul 25 & $08: 58: 25$ & 1.182 & 1.1 & 120 & 90 & $4 \times 30$ \\
\hline GJ1019 & $00: 43: 35.52$ & $+26: 28: 25.1$ & M4 & 14.52 & 19.27 & 2015 Oct 14 & $05: 33: 38$ & 1.886 & 1.1 & 120 & 90 & $4 \times 30$ \\
\hline NN3056 & 00:47:08.22 & $-23: 30: 31.1$ & M3 & 14.40 & 18.52 & 2015 Aug 13 & $05: 47: 17$ & 1.169 & 1.6 & 120 & 90 & $4 \times 30$ \\
\hline GJ1024 & 00:56:39.19 & $+17: 27: 30.3$ & M4 & 13.71 & 17.42 & 2015 Jul 25 & $08: 49: 12$ & 1.371 & 1.1 & 120 & 90 & $4 \times 30$ \\
\hline GJ1029 & 01:05:39.95 & $+28: 29: 31.3$ & M5 & 14.80 & 12.56 & 2015 Oct 14 & 06:01:32 & 1.919 & 1.2 & 120 & 90 & $4 \times 30$ \\
\hline GJ1031 & 01:08:19.12 & $-28: 48: 23.6$ & M3 & 13.42 & 13.16 & 2015 Aug 13 & 05:33:08 & 1.277 & 1.2 & 120 & 90 & $4 \times 30$ \\
\hline GJ2021 & 01:09:19.02 & $-24: 30: 28.3$ & M4 & 14.52 & 15.38 & 2015 Aug 13 & $05: 40: 14$ & 1.262 & 1.3 & 120 & 90 & $4 \times 30$ \\
\hline NN3076 & 01:11:25.65 & $+15: 26: 18.5$ & M5 & 14.36 & 8.47 & 2015 Jul 25 & $07: 50: 46$ & 1.510 & 1.2 & 120 & 90 & $4 \times 30$ \\
\hline NN3119 & 01:51:04.76 & $-06: 07: 10.0$ & M4.5 & 14.60 & 10.00 & 2015 Jul 25 & $09: 23: 14$ & 1.089 & 1.0 & 120 & 90 & $4 \times 30$ \\
\hline Gl83.1 & 02:00:14.15 & $+13: 02: 38.3$ & M4 & 12.28 & 4.47 & 2015 Jul 25 & 08:03:38 & 1.631 & 1.2 & 120 & 90 & $4 \times 30$ \\
\hline NN3129 & 02:02:44.89 & $+13: 34: 30.9$ & M4.5 & 14.27 & 17.24 & $2015 \mathrm{Jul} 25$ & 08:19:09 & 1.570 & 1.2 & 120 & 90 & $4 \times 30$ \\
\hline NN3142 & $02: 12: 55.31$ & $+00: 00: 17.3$ & M4 & 13.50 & 16.67 & $2015 \mathrm{Jul} 25$ & $09: 33: 35$ & 1.155 & 0.9 & 120 & 90 & $4 \times 30$ \\
\hline NN3143 & $02: 15: 34.75$ & $+33: 57: 34.9$ & M3.5 & 13.58 & 17.24 & 2015 Oct 13 & $05: 53: 42$ & 1.929 & 1.0 & 120 & 90 & $4 \times 30$ \\
\hline NN3149 & $02: 16: 35.90$ & $-30: 58: 05.4$ & M4 & 13.00 & 11.36 & 2015 Aug 13 & $06: 10: 58$ & 1.409 & 1.0 & 120 & 90 & $4 \times 30$ \\
\hline NN3148 & 02:16:41.99 & $-30: 59: 15.8$ & M3 & 12.00 & 11.36 & 2015 Aug 13 & 06:03:07 & 1.453 & 1.0 & 120 & 90 & $4 \times 30$ \\
\hline Gl102 & $02: 33: 37.24$ & $+24: 55: 27.3$ & M3.5 & 12.96 & 9.77 & 2015 Jul 25 & 09:50:28 & 1.651 & 0.8 & 120 & 90 & $4 \times 30$ \\
\hline GJ1055 & 03:09:00.59 & $+10: 01: 16.4$ & M5 & 14.85 & 11.88 & 2015 Dec 15 & 04:41:59 & 1.536 & 0.8 & 120 & 90 & $4 \times 30$ \\
\hline NN3224 & $03: 25: 42.18$ & $+05: 51: 50.4$ & M4.5 & 14.70 & 12.99 & 2015 Dec 15 & 04:50:56 & 1.417 & 0.8 & 120 & 90 & $4 \times 30$ \\
\hline NN3225 & 03:26:45.08 & $+19: 14: 39.3$ & M4.5 & 14.96 & 16.67 & 2015 Oct 11 & 09:23:10 & 1.855 & 1.0 & 120 & 90 & $4 \times 30$ \\
\hline NN3227 & $03: 28: 49.89$ & $+26: 29: 10.3$ & M4 & 13.40 & 18.18 & 2015 Oct 11 & 08:01:56 & 1.689 & 0.4 & 120 & 90 & $4 \times 30$ \\
\hline NN3237 & 03:36:41.04 & $+03: 29: 17.5$ & M5 & 13.86 & 14.29 & 2015 Dec 15 & 04:59:38 & 1.369 & 0.8 & 120 & 90 & $4 \times 30$ \\
\hline NN3253 & 03:52:42.29 & $+17: 00: 55.0$ & M5 & 13.70 & 9.62 & 2015 Oct 14 & 06:27:09 & 1.360 & 0.8 & 120 & 90 & $4 \times 30$ \\
\hline NN3261 & 04:05:38.91 & $+05: 44: 40.3$ & M3.5 & 12.89 & 15.87 & 2015 Dec 15 & 05:07:15 & 1.325 & 0.8 & 120 & 90 & $4 \times 30$ \\
\hline NN3296 & $04: 33: 34.62$ & $+20: 44: 41.7$ & M5 & 14.60 & 15.63 & 2015 Oct 14 & $07: 22: 20$ & 1.432 & 1.2 & 120 & 90 & $4 \times 30$ \\
\hline NN3304 & $04: 38: 13.13$ & $+28: 12: 58.7$ & M4.5 & 12.53 & 10.00 & 2015 Oct 13 & $07: 31: 23$ & 1.665 & 1.0 & 120 & 90 & $4 \times 30$ \\
\hline NN3322 & 05:01:58.86 & $+09: 58: 57.7$ & M3.5 & 11.47 & 7.04 & 2015 Dec 17 & 05:47:09 & 1.367 & 0.7 & 120 & 90 & $4 \times 30$ \\
\hline NN3326 & 05:04:14.67 & $+11: 03: 27.3$ & M5 & 13.75 & 13.70 & 2015 Dec 17 & $05: 58: 45$ & 1.419 & 0.6 & 120 & 90 & $4 \times 30$ \\
\hline NN3332 & 05:06:49.54 & $-21: 35: 04.8$ & M3 & 11.66 & 12.05 & 2015 Oct 21 & $06: 26: 10$ & 1.063 & 1.1 & 60 & 45 & $4 \times 15$ \\
\hline NN3344 & 05:16:00.36 & $-72: 13: 59.7$ & M2 & 11.70 & 19.23 & 2015 Oct 21 & $06: 35: 03$ & 1.523 & 0.8 & 120 & 90 & $4 \times 30$ \\
\hline G1207.1 & $05: 33: 44.55$ & $+01: 56: 39.5$ & M3 & 11.53 & 15.08 & 2015 Dec 17 & $05: 37: 45$ & 1.163 & 0.6 & 60 & 45 & $4 \times 10$ \\
\hline GJ1083 & $05: 40: 25.91$ & $+24: 48: 02.3$ & M7 & 14.85 & 10.34 & 2015 Oct 11 & 08:28:00 & 1.560 & 1.3 & 120 & 90 & $4 \times 30$ \\
\hline NN3405 & $06: 42: 13.46$ & $+03: 35: 26.5$ & M4 & 13.33 & 15.63 & 2015 Dec 17 & $06: 29: 22$ & 1.159 & 0.7 & 120 & 90 & $4 \times 30$ \\
\hline GJ1093 & $06: 59: 29.95$ & $+19: 20: 41.0$ & M5 & 14.83 & 7.76 & 2015 Oct 11 & $08: 48: 44$ & 1.542 & 1.0 & 120 & 90 & $4 \times 30$ \\
\hline NN3423 & 07:03:23.25 & $+34: 41: 54.9$ & M4 & 13.17 & 13.33 & 2015 Nov 06 & $08: 31: 36$ & 1.957 & 1.1 & 120 & 90 & $4 \times 30$ \\
\hline GJ1096 & 07:16:18.07 & $+33: 09: 03.8$ & M5 & 14.48 & 14.90 & 2015 Nov 07 & 08:40:59 & 1.873 & 1.1 & 120 & 90 & $4 \times 30$ \\
\hline Gl268.3 & 07:16:19.93 & $+27: 08: 29.8$ & M3 & 10.85 & 7.94 & 2015 Oct 11 & 08:59:03 & 1.842 & 0.8 & 120 & 90 & $4 \times 10$ \\
\hline NN3454 & $07: 36: 25.38$ & $+07: 04: 38.6$ & M4.5 & 13.22 & 6.17 & 2015 Dec 17 & $06: 37: 36$ & 1.174 & 0.6 & 120 & 90 & $4 \times 30$ \\
\hline NN3463 & 07:51:51.87 & $+05: 32: 51.1$ & M4.5 & 14.75 & 15.92 & 2015 Dec 07 & 07:59:18 & 1.165 & 0.7 & 120 & 90 & $4 \times 30$ \\
\hline GJ1103 & 07:51:54.99 & $-00: 00: 23.2$ & M4.5 & 13.50 & 8.79 & 2015 Dec 07 & 08:26:09 & 1.132 & 0.8 & 120 & 90 & $4 \times 30$ \\
\hline NN3466 & 07:54:55.22 & $-29: 21: 04.4$ & M4 & 13.38 & 12.50 & 2015 Oct 21 & 07:08:15 & 1.462 & 0.8 & 120 & 90 & $4 \times 30$ \\
\hline GJ1108 & 08:08:55.43 & $+32: 49: 02.6$ & $\mathrm{M} 2.8+\mathrm{M} 3.3$ & 12.12 & 17.24 & 2015 Dec 06 & $08: 05: 23$ & 1.855 & 0.9 & 120 & 90 & $4 \times 20$ \\
\hline Gl300 & 08:12:40.98 & $-21: 33: 18.1$ & M3.5 & 12.10 & 5.88 & 2015 Oct 21 & $06: 57: 12$ & 1.734 & 0.7 & 120 & 90 & $4 \times 30$ \\
\hline
\end{tabular}


Table 2

(Continued)

\begin{tabular}{|c|c|c|c|c|c|c|c|c|c|c|c|c|}
\hline Name & R.A. & Decl. & $\begin{array}{l}\text { Spectral } \\
\text { Type }\end{array}$ & $M_{V}$ & $\begin{array}{l}\text { Distance } \\
\quad(\mathrm{pc})\end{array}$ & $\begin{array}{l}\text { Observation } \\
\text { Date }\end{array}$ & $\begin{array}{l}\text { Observation } \\
\text { UT Time }\end{array}$ & Airmass & $\begin{array}{c}\text { Seeing } \\
\left({ }^{\prime \prime}\right)\end{array}$ & $\begin{array}{l}\text { UVB Exposure } \\
\text { (s) }\end{array}$ & $\begin{array}{l}\text { VIS Exposure } \\
\text { (s) }\end{array}$ & $\begin{array}{l}\text { NIR Exposure } \\
\text { (s) }\end{array}$ \\
\hline NN3503 & $08: 31: 22.82$ & $-10: 29: 59.9$ & M4 & 15.00 & 15.38 & 2015 Oct 21 & $07: 25: 13$ & 1.802 & 1.1 & 120 & 90 & $4 \times 30$ \\
\hline GJ2069 & $08: 31: 37.48$ & $+19: 23: 37.5$ & M5 & 11.89 & 8.77 & 2015 Nov 19 & $07: 33: 41$ & 1.596 & 1.0 & 120 & 90 & $4 \times 30$ \\
\hline Gl324 & $08: 52: 40.41$ & $+28: 18: 55.5$ & M4 & 13.14 & 13.09 & 2015 Dec 09 & $07: 47: 06$ & 1.683 & 0.8 & 120 & 90 & $4 \times 30$ \\
\hline NN3518 & $08: 55: 19.62$ & $-23: 52: 14.1$ & M4 & 14.00 & 12.20 & 2015 Oct 21 & $07: 32: 49$ & 1.776 & 0.9 & 120 & 90 & $4 \times 30$ \\
\hline GJ1116 & 08:58:14.39 & $+19: 45: 46.1$ & M5.5 & 14.06 & 5.23 & 2015 Nov 19 & $07: 14: 53$ & 1.883 & 1.0 & 120 & 90 & $4 \times 30$ \\
\hline NN3543 & $09: 16: 20.35$ & $-18: 37: 31.3$ & M2 & 10.75 & 12.50 & 2015 Apr 15 & 00:09:21 & 1.008 & 0.5 & 120 & 70 & $4 \times 10$ \\
\hline NN3549 & $09: 18: 41.36$ & $+26: 45: 46.4$ & M5 & 16.00 & 20.00 & 2015 Dec 09 & $08: 12: 52$ & 1.625 & 1.0 & 120 & 90 & $4 \times 30$ \\
\hline Gl347 & $09: 28: 55.54$ & $-07: 22: 22.0$ & M4.5 & 15.00 & 16.72 & 2015 May 19 & 01:03:31 & 1.332 & 1.2 & 120 & 90 & $4 \times 30$ \\
\hline Gl359 & 09:41:02.70 & $+22: 01: 21.0$ & M4.5 & 14.23 & 12.17 & 2015 Apr 09 & $23: 55: 43$ & 1.557 & 0.8 & 120 & 90 & $4 \times 30$ \\
\hline NN3571 & 09:53:54.82 & $+20: 56: 52.2$ & M4 & 14.05 & 10.20 & 2015 Apr 14 & $23: 41: 40$ & 1.548 & 0.4 & 120 & 90 & $4 \times 30$ \\
\hline NN3572 & 09:55:43.61 & $+35: 21: 41.7$ & M3.5 & 12.73 & 17.54 & 2016 Feb 04 & $07: 13: 20$ & 2.241 & 0.8 & 120 & 90 & $4 \times 30$ \\
\hline NN3573 & 09:56:26.53 & $+22: 38: 57.9$ & M4 & 14.20 & 16.13 & 2015 Apr 12 & $01: 24: 52$ & 1.470 & 0.7 & 120 & 90 & $4 \times 30$ \\
\hline NN3590 & $10: 15: 06.93$ & $+31: 25: 08.7$ & M4 & 13.60 & 18.18 & 2015 Dec 14 & $08: 15: 25$ & 1.909 & 0.7 & 120 & 90 & $4 \times 30$ \\
\hline NN3647 & $11: 11: 51.74$ & $+32: 33: 11.4$ & M3.5 & 12.38 & 12.20 & 2016 Feb 03 & 08:53:00 & 2.250 & 0.8 & 120 & 90 & $4 \times 30$ \\
\hline NN3654 & $11: 16: 37.08$ & $-27: 57: 30.5$ & M3.5 & 13.70 & 15.63 & 2015 May 17 & $02: 22: 37$ & 1.126 & 0.9 & 120 & 90 & $4 \times 30$ \\
\hline NN3657 & $11: 23: 07.96$ & $+25: 53: 36.8$ & M5 & 15.14 & 17.33 & 2016 Feb 05 & $05: 55: 29$ & 1.668 & 0.8 & 120 & 90 & $4 \times 30$ \\
\hline NN3668 & $11: 31: 08.78$ & $-14: 57: 41.2$ & M5 & 14.29 & 12.82 & $2015 \mathrm{Jul} 15$ & $23: 42: 57$ & 1.388 & 0.7 & 120 & 90 & $4 \times 30$ \\
\hline NN3682 & $11: 43: 23.43$ & $+25: 18: 13.5$ & M4 & 13.83 & 18.87 & 2015 May 19 & $00: 52: 43$ & 1.551 & 1.1 & 120 & 90 & $4 \times 30$ \\
\hline NN3685 & $11: 47: 40.46$ & $+00: 15: 19.7$ & M4 & 13.25 & 15.63 & 2015 Jul 15 & $23: 50: 34$ & 1.520 & 0.9 & 120 & 90 & $4 \times 30$ \\
\hline GJ1154 & $12: 14: 15.60$ & $+00: 37: 22.9$ & M4.5 & 13.73 & 8.46 & 2015 Jul 16 & $00: 00: 32$ & 1.431 & 1.0 & 120 & 90 & $4 \times 30$ \\
\hline NN3780 & $13: 23: 37.34$ & $-25: 54: 47.8$ & M3.5 & 12.90 & 12.66 & 2015 Aug 10 & $23: 35: 04$ & 1.256 & 1.2 & 120 & 90 & $4 \times 30$ \\
\hline GJ1179 & $13: 48: 11.82$ & $+23: 36: 50.9$ & M5 & 15.32 & 11.99 & 2015 Jul 16 & 00:08:44 & 1.595 & 0.9 & 120 & 90 & $4 \times 30$ \\
\hline NN3808 & $13: 48: 48.66$ & $+04: 06: 00.9$ & M4 & 14.34 & 16.39 & 2015 Aug 13 & $23: 26: 41$ & 1.417 & 1.3 & 120 & 90 & $4 \times 30$ \\
\hline Gl540.2 & $14: 13: 04.24$ & $-12: 01: 31.5$ & M5 & 13.86 & 11.63 & 2015 Aug 13 & $23: 36: 29$ & 1.192 & 0.9 & 120 & 90 & $4 \times 30$ \\
\hline NN3856 & $14: 32: 11.01$ & $+16: 00: 49.1$ & M5 & 13.61 & 14.93 & 2015 Apr 21 & $06: 55: 55$ & 1.465 & 0.8 & 120 & 90 & $4 \times 30$ \\
\hline GJ1186 & $14: 53: 37.31$ & $+11: 34: 02.2$ & M4.5 & 15.29 & 18.55 & 2015 Apr 21 & $07: 42: 35$ & 1.463 & 0.9 & 120 & 90 & $4 \times 30$ \\
\hline NN3900 & $15: 19: 11.00$ & $-12: 45: 08.2$ & M4 & 12.58 & 13.33 & 2015 Jun 12 & 03:50:18 & 1.065 & 0.9 & 120 & 90 & $4 \times 30$ \\
\hline NN3937 & $16: 04: 20.00$ & $-06: 16: 57.8$ & M4.5 & 15.51 & 16.56 & 2015 Apr 21 & 08:12:07 & 1.120 & 0.7 & 120 & 90 & $4 \times 30$ \\
\hline GJ1204 & $16: 36: 05.18$ & $+08: 48: 47.7$ & M4 & 13.80 & 15.34 & 2015 Jun 12 & 04:52:04 & 1.234 & 0.8 & 120 & 90 & $4 \times 30$ \\
\hline NN3967 & $16: 40: 06.23$ & $+00: 42: 16.9$ & M5 & 13.69 & 11.20 & 2015 Jun 12 & 04:09:01 & 1.106 & 0.8 & 120 & 90 & $4 \times 30$ \\
\hline NN3981 & $16: 58: 24.94$ & $+13: 58: 11.5$ & M4 & 13.13 & 12.99 & 2015 Apr 02 & $09: 25: 52$ & 1.288 & 0.8 & 120 & 90 & $4 \times 30$ \\
\hline GJ1210 & $17: 07: 40.42$ & $+07: 22: 01.7$ & M5 & 14.01 & 12.82 & 2015 Jun 12 & $05: 02: 54$ & 1.192 & 0.7 & 120 & 90 & $4 \times 30$ \\
\hline NN4032 & 17:53:00.42 & $+16: 54: 59.3$ & M3.5 & 12.69 & 17.54 & 2015 Apr 21 & $07: 20: 12$ & 1.432 & 0.9 & 120 & 90 & $4 \times 30$ \\
\hline NN4071 & $18: 42: 45.07$ & $+13: 54: 22.0$ & M5 & 12.81 & 10.42 & 2015 Apr 21 & $07: 32: 02$ & 1.491 & 0.8 & 120 & 90 & $4 \times 30$ \\
\hline G1729 & $18: 49: 50.13$ & $-23: 50: 14.4$ & M3.5 & 10.46 & 2.93 & 2015 Jun 12 & 04:42:07 & 1.067 & 0.8 & 120 & 30 & $4 \times 10$ \\
\hline Gl781.1 & 20:07:45.27 & $-31: 45: 24.9$ & M4 & 12.50 & 19.72 & 2015 Jun 17 & 10:09:34 & 1.307 & 0.8 & 120 & 90 & $4 \times 30$ \\
\hline G1791.2 & 20:29:49.07 & $+09: 41: 23.1$ & M4.5 & 13.05 & 8.76 & 2015 Jun 17 & 09:44:17 & 1.473 & 0.6 & 120 & 90 & $4 \times 30$ \\
\hline G1828 & $21: 26: 53.22$ & $-44: 48: 44.6$ & M3.5 & 14.10 & 14.93 & 2015 Jul 13 & 04:56:12 & 1.155 & 1.1 & 360 & 300 & $4 \times 100$ \\
\hline NN4201 & $21: 32: 22.36$ & $+24: 33: 42.0$ & M4 & 12.66 & 12.35 & 2015 May 28 & 09:50:41 & 1.530 & 0.3 & 120 & 90 & $4 \times 30$ \\
\hline G1836 & 21:39:02.08 & $-24: 09: 40.8$ & M4 & 13.43 & 13.95 & 2015 Jul 13 & $05: 11: 28$ & 1.097 & 0.8 & 360 & 300 & $4 \times 60$ \\
\hline NN4215 & 21:44:08.31 & $+17: 04: 38.2$ & M4.5 & 14.81 & 17.54 & 2015 Jun 15 & 09:49:04 & 1.387 & 0.9 & 120 & 90 & $4 \times 30$ \\
\hline NN4231 & $21: 52: 10.59$ & $+05: 37: 33.7$ & M2.4 & 12.11 & 15.63 & 2015 May 22 & 09:38:37 & 1.197 & 0.8 & 120 & 90 & $4 \times 30$ \\
\hline NN4239 & $21: 56: 56.63$ & $-01: 54: 00.5$ & M5 & 14.64 & 13.33 & 2015 Jul 13 & $04: 38: 48$ & 1.397 & 1.1 & 360 & 300 & $4 \times 100$ \\
\hline NN4274 & $22: 23: 07.34$ & $-17: 36: 36.2$ & M4.5 & 13.25 & 7.46 & 2015 Sep 09 & $04: 51: 51$ & 1.036 & 0.9 & 120 & 90 & $4 \times 30$ \\
\hline NN4279 & $22: 27: 03.07$ & $+06: 49: 33.4$ & M3.5 & 13.22 & 13.89 & 2015 Sep 09 & 05:01:00 & 1.222 & 1.0 & 120 & 90 & $4 \times 30$ \\
\hline NN4282 & $22: 33: 22.92$ & $-09: 36: 53.0$ & M3 & 12.41 & 16.95 & 2015 Sep 09 & 05:09:45 & 1.076 & 0.8 & 120 & 90 & $4 \times 30$ \\
\hline NN4292 & $22: 43: 23.71$ & $+22: 08: 17.8$ & M5 & 15.00 & 15.87 & $2015 \mathrm{Jul} 25$ & $07: 26: 48$ & 1.463 & 1.3 & 120 & 90 & $4 \times 30$ \\
\hline
\end{tabular}


Note. Observed M dwarfs, ordered by R.A. spectral types are from SIMBAD (Wenger et al. 2000). The distances are from the Gliese catalog.

(This table is available in machine-readable form.) 


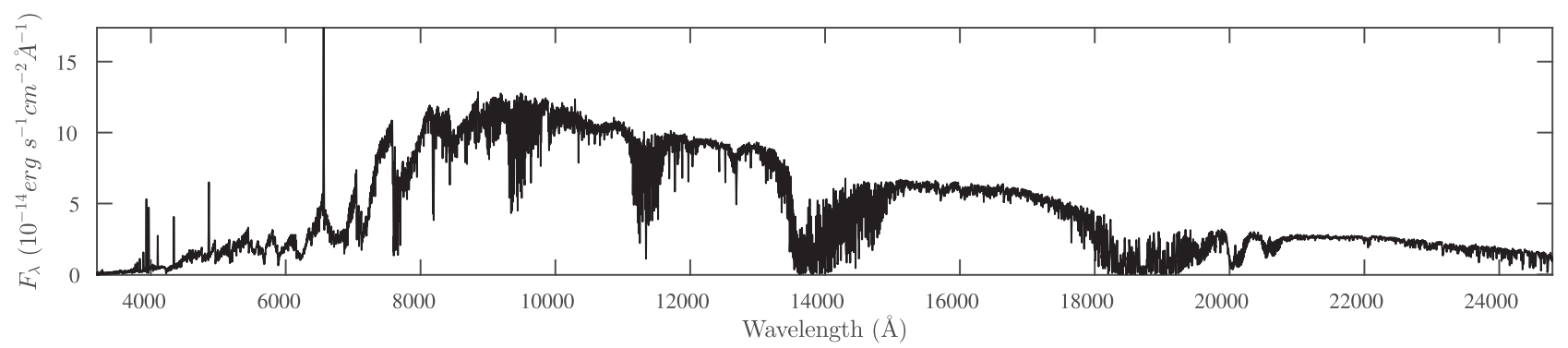

Figure 7. Example of the spectrum of close binary NN3010.

(The complete figure set (26 images) is available.)

\section{ORCID iDs}

Paul Vreeswijk (i) https://orcid.org/0000-0002-7572-9088

Avishay Gal-Yam (10) https://orcid.org/0000-0002-3653-5598

Eran O. Ofek (i) https://orcid.org/0000-0002-6786-8774

\section{References}

Bergeron, P., Leggett, S. K., \& Ruiz, M. T. 2001, ApJS, 133, 413

Bergeron, P., Wesemael, F., Dufour, P., et al. 2011, ApJ, 737, 28

Chen, Y.-P., Trager, S. C., Peletier, R. F., et al. 2014, A\&A, 565, A117

De Rosa, R. J., Patience, J., Wilson, P. A., et al. 2014, MNRAS, 437, 1216

Dong, S., Katz, B., Kushnir, D., \& Prieto, J. L. 2015, MNRAS, 454, L61

Farihi, J., Hoard, D. W., \& Wachter, S. 2010, ApJS, 190, 275

Ferrario, L. 2012, MNRAS, 426, 2500

Fontaine, G., Brassard, P., \& Bergeron, P. 2001, PASP, 113, 409

Freudling, W., Romaniello, M., Bramich, D. M., et al. 2013, A\&A, 559, A96

García-Berro, E., \& Oswalt, T. D. 2016, NewAR, 72, 1

Giammichele, N., Bergeron, P., \& Dufour, P. 2012, ApJS, 199, 29

Gliese, W., \& Jahreiss, H. 1995, yCat, 5070, 0

Holberg, J. B., \& Bergeron, P. 2006, AJ, 132, 1221

Holberg, J. B., Oswalt, T. D., \& Sion, E. M. 2002, ApJ, 571, 512

Holberg, J. B., Sion, E. M., Oswalt, T., et al. 2008, AJ, 135, 1225

Horne, K. 1986, PASP, 98, 609

Howell, D. A. 2011, NatCo, 2, 350

Husser, T.-O., Wende-von Berg, S., Dreizler, S., et al. 2013, A\&A, 553, A6

Jones, D. O., \& West, A. A. 2016, ApJ, 817, 1

Katz, B., \& Dong, S. 2012, arXiv: 1211.4584

Katz, B., Dong, S., \& Kushnir, D. 2014, arXiv:1402.7083

Kepler, S. O., Kleinman, S. J., Nitta, A., et al. 2007, MNRAS, 375, 1315

Kleinman, S. J., Kepler, S. O., Koester, D., et al. 2013, ApJS, 204, 5

Koester, D. 2010, MmSAI, 81, 921
Kowalski, P. M., \& Saumon, D. 2006, ApJL, 651, L137

Kushnir, D., Katz, B., Dong, S., Livne, E., \& Fernández, R. 2013, ApJL, 778, L37

Maoz, D., Mannucci, F., \& Nelemans, G. 2014, ARA\&A, 52, 107

Martin, D. C., Fanson, J., Schiminovich, D., et al. 2005, ApJL, 619, L1

Mason, B. D., Hartkopf, W. I., Gies, D. R., Henry, T. J., \& Helsel, J. W. 2009, AJ, 137, 3358

Mason, B. D., Wycoff, G. L., Hartkopf, W. I., Douglass, G. G., \& Worley, C. E. 2001, AJ, 122, 3466

Moehler, S., Modigliani, A., Freudling, W., et al. 2014, A\&A, 568, A9

Nugent, P. E., Sullivan, M., Cenko, S. B., et al. 2011, Natur, 480, 344

Ofek, E. O. 2014, MATLAB package for astornomy and astrophysics, Astrophysics Source Code Library, ascl:1407.005

Oswalt, J. B. H. T. D., Sion, E. M., \& McCook, G. P. 2016, arXiv:1606.01236

Pickles, A. J. 1998, PASP, 110, 863

Provencal, J. L., Shipman, H. L., Høg, E., \& Thejll, P. 1998, ApJ, 494, 759

Raghavan, D., McAlister, H. A., Henry, T. J., et al. 2010, ApJS, 190, 1

Reid, I. N., \& Hawley, S. L. (ed.) 2005, New Light on Dark Stars Red Dwarfs, Low-Mass Stars, Brown Stars (Chichester: Praxis Publishing Ltd)

Shkolnik, E. L., Hebb, L., Liu, M. C., Reid, I. N., \& Collier Cameron, A. 2010, ApJ, 716, 1522

Sion, E. M., Holberg, J. B., Oswalt, T. D., McCook, G. P., \& Wasatonic, R. 2009, AJ, 138, 1681

Skrutskie, M. F., Cutri, R. M., Stiening, R., et al. 2006, AJ, 131, 1163

Stauffer, J., Tanner, A. M., Bryden, G., et al. 2010, PASP, 122, 885

Tremblay, P.-E., Bergeron, P., \& Gianninas, A. 2011, ApJ, 730, 128

Vernet, J., Dekker, H., D’Odorico, S., et al. 2011, A\&A, 536, A105

Weidemann, V. 2000, A\&A, 363, 647

Wenger, M., Ochsenbein, F., Egret, D., et al. 2000, A\&AS, 143, 9

West, A. A., Hawley, S. L., Walkowicz, L. M., et al. 2004, AJ, 128, 426

Winget, D. E., \& van Horn, H. M. 1987, in IAU Coll. 95: Second Conf. Faint Blue Stars (Schnectady, NY: Davis Press), 363

Yaron, O., \& Gal-Yam, A. 2012, PASP, 124, 668 